\title{
Prosodic phrases and syntactic structure in spontaneous speech
}

\author{
JOHN TØNDERING
}

This study examines prosody and the relationship between syntactic and prosodic boundaries in spontaneously spoken Danish (Standard Copenhagen). The investigation is based on non-scripted monologues taken from Danish Phonetically Annotated Spontaneous Speech Corpus (DanPASS). It is shown that the pitch range of the prosodic phrases correlates with the number of stressed syllables in the phrases. Thus, it is observed that the longer the phrase, the lower the pitch offset. An analysis based on the articulation rate of each stress group (syllables per second) shows final lengthening in the prosodic phrases. The last syllable in the phrase — stressed or unstressed — is the primary target of the lengthening. Furthermore, prosodic phrases are often succeeded by a pause. The observations lead to the conclusion that the prosodic phrase in spontaneous Danish is of a different kind than the prosodic phrase in read aloud Danish.

The material has been analysed syntactically, and the prosodic boundaries are compared with the syntactic boundaries. It is concluded that a prosodic phrase break is most frequently placed in a syntactic boundary between two sentences or in an internal syntactic boundary in a complex sentence. From the alternative perspective it is seen that syntactic boundaries delimiting independent clauses are always accompanied with prosodic phrase breaks. Internal syntactic boundaries between independent and dependent clauses are not always marked with a prosodic break. The lowest degree of co-occurring syntactic and prosodic boundaries is seen between matrix and complement clauses. The highest degree of prosodic breaks is seen in boundaries between matrix and adverbial clauses. 